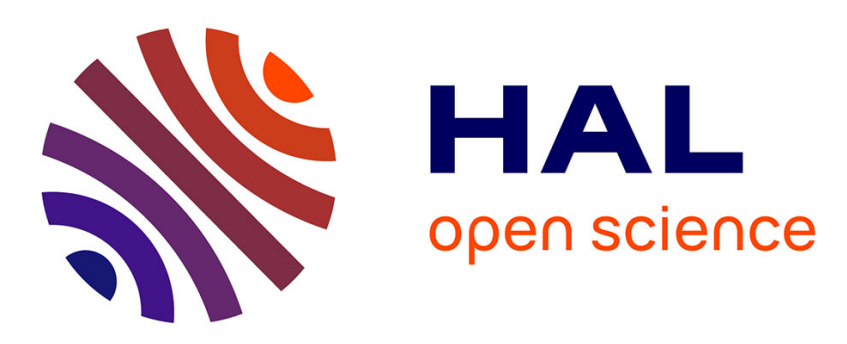

\title{
Subspace based methods for continuous-time model identification of MIMO systems from filtered sampled data
}

Guillaume Mercère, Régis Ouvrard, Marion Gilson, Hugues Garnier

\section{- To cite this version:}

Guillaume Mercère, Régis Ouvrard, Marion Gilson, Hugues Garnier. Subspace based methods for continuous-time model identification of MIMO systems from filtered sampled data. European Control Conference, ECC'07, Jul 2007, Kos, Greece. pp.CDROM. hal-00150100

\section{HAL Id: hal-00150100 \\ https://hal.science/hal-00150100}

Submitted on 29 May 2007

HAL is a multi-disciplinary open access archive for the deposit and dissemination of scientific research documents, whether they are published or not. The documents may come from teaching and research institutions in France or abroad, or from public or private research centers.
L'archive ouverte pluridisciplinaire HAL, est destinée au dépôt et à la diffusion de documents scientifiques de niveau recherche, publiés ou non, émanant des établissements d'enseignement et de recherche français ou étrangers, des laboratoires publics ou privés. 


\title{
Subspace based methods for continuous-time model identification of MIMO systems from filtered sampled data
}

\author{
Guillaume Mercère, Régis Ouvrard, Marion Gilson and Hugues Garnier
}

\begin{abstract}
This article introduces a new identification method for continuous-time MIMO state space models from sampled input output data. The proposed approach consists more precisely in combining filtering techniques with a specific subspace algorithm. Two filtering methods (the reinitialised partial moments and the Poisson moment functionals) are considered to circumvent the time derivative problem inherent in continuoustime modelling. The developed subspace algorithm belongs to the MOESP method family. A particular attention is payed to the construction of the instrumental variable used to supply consistent and accurate estimates in a noisy framework. The benefits of the proposed algorithms in comparison with existing methods are illustrated with a simulation study.
\end{abstract}

\section{INTRODUCTION}

Subspace based identification methods (SIM) have received an increasing attention in the last fifteen years [1], [2]. These techniques have indeed the property to supply a linear state space model of the system from input output $(\mathrm{I} / \mathrm{O})$ data without non linear optimization generally required by the prediction methods [3] with the help of computational tools such as the RQ factorization and the singular value decomposition (SVD). Most of these methods have been developed for discrete-time (DT) models [4], [5]. However, in many applications (modelling, diagnosis, control), it is interesting to deal with a continuous-time (CT) model in order to have access to the physical parameters of the system. To reach this goal, a first indirect approach consists in firstly determining a DT model via conventional identification algorithms [3], then converting this representation in a CT model. The statistical properties in this framework are indeed well known and explain the affinity with this point of view. Unfortunately, this indirect approach suffers from many limitations, as [6]

- the difficult choice of the sampling period,

- the use of the matrix logarithm which may produce complex arithmetic when the matrices have negative eigenvalues,

- the tricky translation of zeros in the CT domain [7].

G. Mercère is with the Laboratoire d'Automatique et d'Informatique Industrielle, EA 1219, 40, avenue du Recteur Pineau, Bâtiment de Mécanique, 86022 Poitiers Cedex, France guillaume.mercereduniv-poitiers.fr

R. Ouvrard is with the Laboratoire d'Automatique et d'Informatique Industrielle, EA 1219, 40, avenue du Recteur Pineau, Bâtiment de Mécanique, 86022 Poitiers Cedex, France regis.ouvrardauniv-poitiers.fr

M. Gilson is with the Centre de Recherche en Automatique de Nancy, Nancy-Université (UMR 7039), CNRS, BP 239, F-54506 Vandoeuvre-lèsNancy Cedex, France marion.gilson@cran. uhp-nancy . fr

H. Garnier is with the Centre de Recherche en Automatique de Nancy, Nancy-Université (UMR 7039), CNRS, BP 239, F-54506 Vandoeuvre-lèsNancy Cedex, France hugues.garnierdcran.uhp-nancy. fr
All these restrictions can be alleviated with the direct approach which consists in directly estimating a CT model from sampled I/O data [6] [8]. The main problem of these techniques is the computation of the successive $\mathrm{I} / \mathrm{O}$ time derivatives. The solution often rests on the use of linear filters, integral methods or modulating functions [9]. In this paper, the reinitialised partial moments [10] and the Poisson moment functionals [11] are more particularly considered. Despite a development in a different framework, these techniques can be rewritten as an I/O filtering approach and supply a solution to the time derivative calculation which is relatively straightforward.

The direct CT estimation methods have essentially been developed for SISO systems. The MIMO framework is comparatively not so developed [12], [13], [14], [15], [16], [17]. In this article, this problem is considered by associating respectively the reinitialised partial moments and the Poisson moment functionals to a particular class of SIM: the MOESP algorithms [18], [19], [20], [21]. These subspace techniques, mainly based on the observability subspace estimation, use orthogonal projections which increases the global numerical robustness. The disturbances problem is treated by introducing a particular instrumental variable for the estimation of the system observability matrix basis.

The paper is organized as follows. Section II introduces the identification problem. Section III briefly presents both techniques used to circumvent the time derivative problem and gives the filtered data equation used in CT subspace identification. Then, the development of two new estimation algorithms is considered in Section IV. The problem of the instrumental variable construction for consistently modelling the system from noisy I/O data is more particularly analyzed. Some practical conditions for the choice of the user parameters are also given. In Section V, the performances of these new techniques are illustrated from numerical simulations.

\section{PROBLEM FORMULATION AND NOTATIONS}

Consider a linear time invariant CT system described by the following state space equations

$$
\begin{aligned}
\dot{\mathbf{x}}(t) & =\mathbf{A x}(t)+\mathbf{B u}(t)+\mathbf{w}(t) \\
\mathbf{y}(t) & =\mathbf{C x}(t)+\mathbf{D u}(t)+\mathbf{v}(t)
\end{aligned}
$$

where $\mathbf{u} \in \mathbb{R}^{n_{u}}$ and $\mathbf{y} \in \mathbb{R}^{n_{y}}$ are respectively the input and output signal, $\mathbf{x} \in \mathbb{R}^{n_{x}}$ the state vector and $\mathbf{w}$ and $\mathbf{v}$ the zero mean process and output measurement stochastic disturbances. Following the same reasoning as in [22], it is supposed that the process and the measurement noise are constant between sampling instances: $\mathbf{w}(t)=\mathbf{w}\left(k T_{s}\right)$ and 
$\mathbf{v}(t)=\mathbf{v}\left(k T_{s}\right)$ for $k T_{s} \leq t<(k+1) T_{s}$, where $T_{s}$ denotes the sampling period. These disturbances are furthermore assumed to be DT white noise sequences with the following correlation

$$
\begin{aligned}
\mathbb{E}\left\{[ \begin{array} { c } 
{ \mathbf { v } ( k T _ { s } ) } \\
{ \mathbf { w } ( k T _ { s } ) }
\end{array} ] \left[\mathbf{v}^{T}\left(l T_{s}\right)\right.\right. & \left.\left.\mathbf{w}^{T}\left(l T_{s}\right)\right]\right\}= \\
& {\left[\begin{array}{cc}
\mathbf{R}_{\mathbf{v}} & \mathbf{R}_{\mathbf{v w}} \\
\mathbf{R}_{\mathbf{v w}}^{T} & \mathbf{R}_{\mathbf{w}}
\end{array}\right] \delta(k, l) }
\end{aligned}
$$

where $\delta(k, l)$ is the Kronecker delta function and $\mathbb{E}\{\bullet\}$ the mathematical expectation. The justification for assuming such noise models for $\mathbf{v}(t)$ and $\mathbf{w}(t)$ are given in [22], [12].

The goal of CT subspace identification is to estimate the state space matrices $\{\mathbf{A}, \mathbf{B}, \mathbf{C}, \mathbf{D}\}$, up to a similarity transformation, from DT I/O data $\left\{\mathbf{u}\left(t_{k}\right), \mathbf{y}\left(t_{k}\right)\right\}_{k=1}^{N}$ with $t_{k}=k T_{s}$.

In order to introduce the CT subspace identification problem and the link with the DT approach, assume firstly that

- the disturbances $\mathbf{v}$ and $\mathbf{w}$ are equal to zero,

- the signals $\mathbf{u}$ and $\mathbf{y}$ are differentiable at least to the $(i-1)$ th time derivative with $i>n_{x}$.

Then, the following yields [15]

$$
\mathbf{y}_{i}\left(t_{k}\right)=\boldsymbol{\Gamma}_{i} \mathbf{x}\left(t_{k}\right)+\mathbf{H}_{i} \mathbf{u}_{i}\left(t_{k}\right)
$$

where $\mathbf{y}_{i}\left(t_{k}\right)$ (resp. $\mathbf{u}_{i}\left(t_{k}\right), \mathbf{w}_{i}\left(t_{k}\right)$ and $\mathbf{v}_{i}\left(t_{k}\right)$ ) is a stacked vector composed by $i-1$ time derivatives of $\mathbf{y}$ (resp. $\mathbf{u}, \mathbf{w}$ and $\mathbf{v})$ at $t=t_{k}$

$$
\mathbf{y}_{i}\left(t_{k}\right)=\left[\begin{array}{llll}
\mathbf{y}^{T}\left(t_{k}\right) & \mathbf{y}^{(1) T}\left(t_{k}\right) & \cdots & \mathbf{y}^{(i-1)^{T}}\left(t_{k}\right)
\end{array}\right]^{T},
$$

$\boldsymbol{\Gamma}_{i}$ is the extended observability matrix

$$
\boldsymbol{\Gamma}_{i}=\left[\begin{array}{llll}
\mathbf{C}^{T} & (\mathbf{C A})^{T} & \cdots & \left(\mathbf{C A}^{i-1}\right)^{T}
\end{array}\right]^{T}
$$

and $\mathbf{H}_{i}$ is the block Toeplitz matrices of the impulse response from $\mathbf{u}$ to $\mathbf{y}$. For $N-k+1$ samples, (3) becomes

$$
\mathbf{Y}_{i}=\boldsymbol{\Gamma}_{i} \mathbf{X}+\mathbf{H}_{i} \mathbf{U}_{i}
$$

with $^{1}$

$$
\mathbf{Y}_{i}=\left[\begin{array}{llll}
\mathbf{y}_{i}\left(t_{k}\right) & \mathbf{y}_{i}\left(t_{k+1}\right) & \cdots & \mathbf{y}_{i}\left(t_{N}\right)
\end{array}\right]
$$

and

$$
\mathbf{X}=\left[\begin{array}{llll}
\mathbf{x}\left(t_{k}\right) & \mathbf{x}\left(t_{k+1}\right) & \cdots & \mathbf{x}\left(t_{N}\right)
\end{array}\right]
$$

Equation (6) has the particularity to be structurally equivalent to the so called data equation used in DT subspace identification [4]. Thus, it seems possible to apply the classical following steps to estimate the system state space matrices [21]

- a compression of the I/O data via an RQ factorization,

- the estimation of a basis of the extended observability matrix from specific submatrices of the resulting lower triangular factor in a consistent way via singular value decomposition (SVD),

\footnotetext{
${ }^{1} \mathbf{U}_{i}$ is built up in a similar way as $\mathbf{Y}_{i}$.
}

- the extraction of the $\mathbf{A}$ and $\mathbf{C}$ matrices via the Ainvariance of $\boldsymbol{\Gamma}_{i}$ and the estimation of $\mathbf{B}$ and $\mathbf{D}$ from a linear regression optimization.

However, this algorithmic procedure is based on an unavoidable assumption: the successive time derivatives of the data have to be measured to obtain equation (6). Unfortunately, in practice, the direct computation of the time derivatives of the $\mathrm{I} / \mathrm{O}$ is seldom feasible, particularly in a noisy framework. It is therefore essential to resort to particular techniques which are able to evaluate these time derivatives from measured sampled data. Many algorithms have been developed to reach this goal during the last three decades (see [9] for an overview). A comparative study based on Monte Carlo simulations has shown that six CT methods present the best performances. Two ${ }^{2}$ of them are considered in the following: the Poisson moment functional (pmf) approach [24] and the reinitialised partial moments (rpm) method [25].

\section{APPROXIMATION OF THE I/O TIME DERIVATIVE DATA}

\section{A. The reinitialised partial moments (rpm)}

The reinitialised partial moments method [10] can be firstly considered as an integration method, i.e. a technique which avoids the differentiation of the data by performing a sufficient order integration. Let $s(t)$ be a function defined on $[0, \infty[$. Then, the $n$th reinitialised partial moment is defined as $^{3}$

$$
M_{n}\{s(t)\}=\int_{0}^{T} \frac{\tau^{n}}{n !} s(t-T+\tau) d \tau .
$$

This integration function is an extension of the classical moment $\int_{0}^{\infty} \tau^{n} s(\tau) d \tau$. The main characteristics of the rpm are to perform a time weighing of the I/O signals and a time shifting before integrating them. Concerning the later, the prediction error estimation variance is minimal for an optimal value of $T=\hat{T}$. In order to take advantage of this property, the integration is reinitialised at each sampling time. Although the determination of $\hat{T}$ is essential, experiences show that the rpm method is not too sensitive to this user parameter. In practice, it can be chosen about twice as the main time constant for an aperiodic system or twice as the rising time for an underdamped system.

Secondly, the rpm method can be introduced from a filtering point of view. Indeed, the integration can be expressed as a time convolution product

$$
\bar{M}_{n}\{s(t)\}=\int_{0}^{\hat{T}} \frac{(\hat{T}-\tau)^{n} \tau^{n-1}}{\hat{T}^{n}(n-1) !} s(t-\tau) d \tau=\left[h_{n} \star s\right](t)
$$

where

$$
\begin{aligned}
& h_{n}(t)=\frac{(\hat{T}-t)^{n} t^{n-1}}{\hat{T}^{n}(n-1) !} \text { for } t \in[0, \hat{T}] \\
& h_{n}(t)=0 \text { elsewhere. }
\end{aligned}
$$

\footnotetext{
${ }^{2}$ Both transformations are available in the Matlab toolbox CONTSID [23] (see http://www.cran.uhp-nancy.fr/contsid/).

${ }^{3} M_{n}\{s(t)\}$ is defined for $t \geq \hat{T}$.
} 
Thus, since the integration window is a time shifting window, the use of the rpm in the considered identification framework amounts to apply a finite impulse response filter to the $\mathrm{I} / \mathrm{O}$ data. This property results in making the initial conditions vanish. Furhermore, for the same reason, the first $\hat{T}$ samples must be removed.

\section{B. The Poisson moment functionals (pmf)}

An alternative solution to handle the time derivative problem is to use the Poisson moment functionals approach which can also be written in terms of filtering. The $n$th order $p m f$ transform of the signal $s(t)$ over $[0, t]$ is indeed given by the following convolution product [11]

$$
M_{n}\{s(t)\}=\int_{0}^{t} g_{n}(\tau) s(t-\tau) d \tau=\left[g_{n} \star s\right](t)
$$

where

- $g_{n}(t)=\beta^{n+1} \frac{t^{n}}{n !} e^{-\lambda t}$ is the $n$th Poisson pulse function,

- $\lambda \in \mathbb{R}^{+}$and $\beta \in \mathbb{R}^{+*}$ are the Poisson filter constant and gain respectively (most of the time, $\beta=\lambda$ is set).

This linear transform consists then in filtering the signal $s(t)$ by the filter $g_{n}(t)$, whose transfer function is given as (Laplace transform of the Poisson impulse function)

$$
\mathcal{L}\left(g_{n}(t)\right)=\left(\frac{\beta}{p+\lambda}\right)^{n+1}
$$

where $p$ is the Laplace variable. The $n$th order Poisson moment functional of $s(t)$ can be considered as the output of a cascaded low-pass filter chain of $n+1$ identical stages, each with a transfer function given by $\beta /(p+\lambda)$. The user parameter is the cut-off frequency of the filter. It should be chosen in order to emphasise the frequency band of interest and it is advised in general to choose it a little bit larger than the bandwidth of the system to be identified. Moreover, it has been shown that the initial conditions can be neglected after a short period of time [9].

\section{Filtered data equation}

The description of these two transformations via a time convolution product allows us to formulate their use for our identification problem in a global way. More precisely, if the impulse response $m_{n}(t)$ respectively stands for

$$
m_{n}(t)=\left\{\begin{array}{l}
h_{n}(t) \text { for the } r p m \\
g_{n}(t) \text { for the pmf }
\end{array}\right.
$$

for all vector $\mathbf{z}_{i}(t) \in \mathbb{R}^{n_{z} i}$ defined as

$$
\mathbf{z}_{i}(t)=\left[\begin{array}{llll}
\mathbf{z}^{T}(t) & \mathbf{z}^{(1)^{T}}(t) & \cdots & \mathbf{z}^{(i-1)^{T}}(t)
\end{array}\right]^{T}
$$

it is straightforward to check that

$$
\begin{gathered}
{\left[\mathbf{z}_{i} \star m_{n}\right](t)=\left[\begin{array}{c}
{\left[\mathbf{z} \star m_{n}\right](t)} \\
{\left[\mathbf{z}^{(1)} \star m_{n}\right](t)} \\
\vdots \\
{\left[\mathbf{z}^{(i-1)} \star m_{n}\right](t)}
\end{array}\right]} \\
=\left[\begin{array}{c}
{\left[\mathbf{z} \star m_{n}\right](t)} \\
{\left[\mathbf{z} \star m_{n}^{(1)}\right](t)} \\
\vdots \\
{\left[\mathbf{z} \star m_{n}^{(i-1)}\right](t)}
\end{array}\right]:=\left[\begin{array}{c}
M_{0}^{n}\{\mathbf{z}(t)\} \\
M_{1}^{n}\{\mathbf{z}(t)\} \\
\vdots \\
M_{i-1}^{n}\{\mathbf{z}(t)\}
\end{array}\right]
\end{gathered}
$$

with $n \geq i+1$ by assuming that ${ }^{4}$

$$
\begin{aligned}
& m_{n}^{(k-1)} \text { holds for } k \in[1, i] \\
& m_{n}^{(k-1)}(t=0)=0 \text { for } k \in[1, i] \\
& m_{n}^{(k-1)}(t=\theta)=0 \text { for } k \in[1, i]
\end{aligned}
$$

where $\theta=\hat{T}$ for the rpm and $\theta=t$ for the pmf. This transformation, which is linked to the properties of the convolution product, allows to transfer the derivative operation on the function $m_{n}$ which is differentiable at order $n$ by construction. The I/O measurement time derivative problem is also circumvented. Thus, equation (6) becomes (in a noisy framework)

$$
\begin{aligned}
\mathcal{M}\left\{\mathbf{Y}_{i}\right\}=\boldsymbol{\Gamma}_{i} \mathcal{M}\{\mathbf{X}\} & +\mathbf{H}_{i} \mathcal{M}\left\{\mathbf{U}_{i}\right\} \\
& +\mathbf{G}_{i} \mathcal{M}\left\{\mathbf{W}_{i}\right\}+\mathcal{M}\left\{\mathbf{V}_{i}\right\}
\end{aligned}
$$

where $\mathcal{M}\{\bullet\}$ denotes the transform applied to sampled data to approximate the $\mathrm{I} / \mathrm{O}$ data time derivative

$$
\mathcal{M}\left\{\mathbf{Y}_{i}\right\}=\left[\begin{array}{ccc}
M_{0}^{n}\left\{\mathbf{y}\left(t_{k}\right)\right\} & \cdots & M_{0}^{n}\left\{\mathbf{y}\left(t_{N}\right)\right\} \\
M_{1}^{n}\left\{\mathbf{y}\left(t_{k}\right)\right\} & \cdots & M_{1}^{n}\left\{\mathbf{y}\left(t_{N}\right)\right\} \\
\vdots & & \vdots \\
M_{i-1}^{n}\left\{\mathbf{y}\left(t_{k}\right)\right\} & \cdots & M_{i-1}^{n}\left\{\mathbf{y}\left(t_{N}\right)\right\}
\end{array}\right]
$$

where

$$
\mathcal{M}\{\mathbf{X}\}=\left[\begin{array}{lll}
M_{0}^{n}\left\{\mathbf{x}\left(t_{k}\right)\right\} & \cdots & M_{0}^{n}\left\{\mathbf{x}\left(t_{N}\right)\right\}
\end{array}\right]
$$

and where $\mathbf{G}_{i}$ is the block Toeplitz matrices of the impulse response from $\mathbf{w}$ to $\mathbf{y}$.

\section{SUBSPACE IDENTIFICATION ALGORITHM}

Consider from now the equation of the filtered data (20). Then, assuming that the input is sufficiently exciting (see [26] for conditions), that the initial condition are zero and that the transformation does not remove any state variable, (i.e. $\operatorname{rank}\{\mathcal{M}\{\mathbf{X}\}\}=n_{x}$ ), it is possible to apply the DT identification algorithms to the CT problem. The MOESP schemes [18], [19], [20], [21] are more precisely considered in the following. The algorithms gathered under this acronym have indeed some interesting numerical advantages due to the use of orthogonal projections (see [27, Chapter 5] for an interesting study).

\footnotetext{
${ }^{4}$ By definition, these properties are verified by the rpm. Furthermore, $g_{n}(0)=0$ by construction. $g_{n}(\theta)$ can be considered as equal to zero if $\theta=t$ is sufficiently big.
} 


\section{A. Identification in a noisy framework}

In a noisy general case ${ }^{5}$, i.e. when both $\mathbf{v}$ and $\mathbf{w}$ are non zero, the DT MOESP algorithms (PI MOESP and PO MOESP [20], [21]) are based on a particular RQ factorization where an instrumental variable is introduced to remove the effects of the noise term. In the CT framework considered in this paper, we suggest to use the following RQ factorization

$$
\left[\begin{array}{c}
\mathcal{M}\left\{\mathbf{U}_{i}\right\} \\
\boldsymbol{\Xi} \\
\mathcal{M}\left\{\mathbf{Y}_{i}\right\}
\end{array}\right]=\left[\begin{array}{ccc}
\mathbf{R}_{11} & \mathbf{0} & \mathbf{0} \\
\mathbf{R}_{21} & \mathbf{R}_{22} & \mathbf{0} \\
\mathbf{R}_{31} & \mathbf{R}_{32} & \mathbf{R}_{33}
\end{array}\right]\left[\begin{array}{l}
\mathbf{Q}_{1} \\
\mathbf{Q}_{2} \\
\mathbf{Q}_{3}
\end{array}\right]
$$

where $^{6} \boldsymbol{\Xi}$ is made of instruments chosen such that the disturbances effects are asymptotically cancelled without modifying the informative part of the signal terms, i.e.

$$
\begin{aligned}
& \lim _{N \rightarrow \infty} \frac{1}{N} \mathcal{M}\left\{\mathbf{V}_{i}\right\} \boldsymbol{\Xi}^{T}=\lim _{N \rightarrow \infty} \frac{1}{N} \mathcal{M}\left\{\mathbf{W}_{i}\right\} \boldsymbol{\Xi}^{T}=\mathbf{0} \\
& \operatorname{rank}\left\{\lim _{N \rightarrow \infty} \frac{1}{N} \mathcal{M}\{\mathbf{X}\} \boldsymbol{\Xi}^{T}\right\}=n_{x} .
\end{aligned}
$$

Indeed, since this RQ factorization gives access to the following relation [4]

$$
\mathbf{R}_{32} \mathbf{Q}_{2}=\mathcal{M}\left\{\mathbf{Y}_{i}\right\} \boldsymbol{\Pi}_{\mathcal{M}\left\{\mathbf{U}_{i}\right\}^{\perp}} \boldsymbol{\Xi}^{T} \mathbf{P}_{d}
$$

where $\mathbf{P}_{d}$ is a particular weighting matrix depending on $\mathcal{M}\left\{\mathbf{U}_{i}\right\}$ and $\boldsymbol{\Xi}$. Then, it is relatively straightforward to show that (see Appendix)

$$
\begin{aligned}
& \lim _{N \rightarrow \infty} \frac{1}{N} \mathbf{R}_{32} \mathbf{Q}_{2}= \\
& \lim _{N \rightarrow \infty} \frac{1}{N} \boldsymbol{\Gamma}_{i} \mathcal{M}\{\mathbf{X}\} \boldsymbol{\Pi}_{\mathcal{M}\left\{\mathbf{U}_{i}\right\}^{\perp}} \boldsymbol{\Xi}^{T} \mathbf{P}_{d}
\end{aligned}
$$

under the assumptions that the noise terms are uncorrelated with the input signal and the instruments. Hence, equation (26) proves that it is possible to estimate a basis of the observability matrix from $\mathbf{R}_{32}$ when

$$
\operatorname{rank}\left\{\mathcal{M}\{\mathbf{X}\} \boldsymbol{\Pi}_{\mathcal{M}\left\{\mathbf{U}_{i}\right\}^{\perp}} \boldsymbol{\Xi}^{T} \mathbf{P}_{d}\right\}=n_{x} .
$$

This basis is obtained by applying a SVD to this block matrix. At the same time, the order estimation can be realised by analyzing the singular values which follow from this decomposition. Some estimation techniques, based on particular information criteria, are available in the literature [28], [5].

\section{B. Choice of the instrumental variable}

The algorithm developed in the previous subsection is based on an essential assumption: the instrumental variable has to be chosen such as equation (24) is fulfilled. In general, in a CT framework, the instruments are built up by increasing the time derivative order $i$ and, by extension, the number of rows of the matrices composing the filtered data equation (20) [13], [14], [16]. For example, R. Johansson

\footnotetext{
${ }^{5}$ The noise free case is not introduced in this article due to the lack of space. This situation can however be considered as a special case of the algorithm developed in the noisy framework.

${ }^{6}$ The problem of the instrumental variable construction is studied in subsection IV-B.
}

et al recommend to choose $i \geq n_{u}+n_{y}+n_{x}$. The main drawback of this approach is that time derivative of high order have to be computed. Moreover, it is well known that using many approximations of time derivative practically leads to numerical performances corruption. Two solutions are therefore presented is the following to overcome this drawback.

1) Use of past inputs as IV: It is firstly suggested to use in a first time shifted version of the sampled input data and build up a particular Hankel matrix

$$
\begin{aligned}
& \boldsymbol{\Xi}=\mathcal{U}_{p}= \\
& {\left[\begin{array}{cccc}
\mathbf{u}\left((k-p) T_{s}\right) & \mathbf{u}\left((k+1-p) T_{s}\right) & \cdots & \mathbf{u}\left((N-p) T_{s}\right) \\
\vdots & \vdots & & \vdots \\
\mathbf{u}\left((k-1) T_{s}\right) & \mathbf{u}\left(k T_{s}\right) & \cdots & \mathbf{u}\left((N-1) T_{s}\right)
\end{array}\right]}
\end{aligned}
$$

as considered in the DT case [20]. A straightforward way to study the efficiency and properties of this instrumental variable consists in analyzing the following limits

$$
\begin{aligned}
& \lim _{N \rightarrow \infty} \frac{1}{N} \mathcal{M}\left\{\mathbf{V}_{i}\right\} \mathcal{U}_{p}^{T} \\
& \lim _{N \rightarrow \infty} \frac{1}{N} \mathcal{M}\left\{\mathbf{W}_{i}\right\} \mathcal{U}_{p}^{T} .
\end{aligned}
$$

Since $\mathbf{v}$ and $\mathbf{w}$ have the same characteristics, only equation (29a) is used. Let us consider the following $q$ th block row and $r$ th block column entry of $\frac{1}{N} \mathcal{M}\left\{\mathbf{V}_{i}\right\} \mathcal{U}_{p}^{T}$

$$
\begin{aligned}
& \frac{1}{N}\left(\mathcal{M}\left\{\mathbf{V}_{i}\right\} \mathcal{U}_{p}^{T}\right)_{q r}= \\
& \frac{1}{N} \sum_{j=1}^{N} M_{q}^{n}\left\{\mathbf{v}\left(t_{j}\right)\right\} \mathbf{u}^{T}\left((j+r-p) T_{s}\right) .
\end{aligned}
$$

This leads to

$$
\begin{aligned}
\frac{1}{N}\left(\mathcal{M}\left\{\mathbf{V}_{i}\right\} \mathcal{U}_{p}^{T}\right)_{q r} & =\int_{0}^{\theta} m_{n}^{(q)}(\tau) \\
& \left(\frac{1}{N} \sum_{j=k}^{N} \mathbf{v}\left(j T_{s}-\tau\right) \mathbf{u}^{T}\left((j+r-p) T_{s}\right)\right) d \tau
\end{aligned}
$$

where $m_{n}^{(q)}$ is defined as in (14) according to the chosen filtering method and

$$
\begin{aligned}
& \theta=\hat{T} \text { for the } r p m \\
& \theta=t \text { for the pmf. }
\end{aligned}
$$

By assuming that

- the input and noise signals are ergodic processes,

- the system operates in open loop,

it holds that

$$
\lim _{N \rightarrow \infty} \frac{1}{N} \sum_{j=k}^{N} \mathbf{v}\left(j T_{s}-\tau\right) \mathbf{u}^{T}\left((j+r-p) T_{s}\right)=\mathbf{0} .
$$

Hence, since each component of $\lim _{N \rightarrow \infty} \frac{1}{N} \mathcal{M}\left\{\mathbf{V}_{i}\right\} \mathcal{U}_{p}^{T}$ tends to $\mathbf{0}$ when $N \rightarrow \infty$, equation (24a) is satisfied. 
Concerning the value of the user parameter $p$, it has been shown in the DT framework [4], [26] that choosing $p \geq \frac{n_{x}}{n_{u}}$ guarantees that

$$
\lim _{N \rightarrow \infty} \frac{1}{N} \mathcal{X}_{f} \boldsymbol{\Pi}_{\mathcal{U}_{f}^{\perp}} \mathcal{U}_{p}^{T} \text { exists and has full rank } n_{x},
$$

with $\mathcal{U}_{f}$ the Hankel matrix of "future" sampled inputs and $\mathcal{X}_{f}$ composed by "future" state vectors. Although the CT problem considers filtered versions of the input and the state signals, the condition $p \geq \frac{n_{x}}{n_{u}}$ is a necessary requirement to have the number of rows of $\mathcal{U}_{p}$ at least equal to $n_{x}$. Thus, since the instrumental variable is made up with the same reasoning as in the DT case, this condition on $p$ can be used in our CT identification problem. An easy way to choose $p$ consists, for example, in using $p=i$ since $i>n_{x}$.

2) Use of past outputs as IV: In the DT framework, it is often suggested to introduce past output data among the instruments in order to get more accurate estimates [21]. Thus, it is interesting to analyse the effect of the use of $\mathcal{Y}_{p}$ as instrumental variable. First of all, consider the following relation

$$
\mathcal{Y}_{p}=\boldsymbol{\Gamma}_{p} \mathcal{X}_{p}+\mathbf{H}_{p} \mathcal{U}_{p}+\mathbf{G}_{p} \mathcal{V}_{p}+\mathcal{W}_{p}
$$

where $\boldsymbol{\Gamma}_{p}, \mathbf{H}_{p}$ and $\mathbf{G}_{p}$ are defined as previously with $i=p$, where $\mathcal{Y}_{p}, \mathcal{V}_{p}$ and $\mathcal{V}_{p}$ are built up as $\mathcal{U}_{p}$ and where

$$
\boldsymbol{\mathcal { X }}_{p}=\left[\begin{array}{lll}
\mathbf{x}\left((k-p) T_{s}\right) & \cdots & \mathbf{x}\left((N-p) T_{s}\right)
\end{array}\right] .
$$

Then, we have ${ }^{7}$

$$
\begin{aligned}
& \frac{1}{N} \mathcal{M}\left\{\mathbf{V}_{i}\right\} \mathcal{Y}_{p}^{T}= \\
& \frac{1}{N}\left(\mathcal{M}\left\{\mathbf{V}_{i}\right\} \mathcal{X}_{p}^{T} \Gamma_{p}^{T}+\mathcal{M}\left\{\mathbf{V}_{i}\right\} \mathcal{U}_{p}^{T} \mathbf{H}_{p}^{T}\right. \\
&\left.+\mathcal{M}\left\{\mathbf{V}_{i}\right\} \mathcal{V}_{p}^{T} \mathbf{G}_{p}^{T}+\mathcal{M}\left\{\mathbf{V}_{i}\right\} \mathcal{W}_{p}^{T}\right) .
\end{aligned}
$$

By assuming that the input and noise signals are uncorrelated, the second term of the right-and-side (rhs) of (37) vanishes as $N$ tends to infinity. The third and fourth elements of the rhs of (37) can be studied in the same way. Consider e.g. $\frac{1}{N} \mathcal{M}\left\{\mathbf{V}_{i}\right\} \mathcal{V}_{p}^{T}$. Then, following the same approach as used previously, its $q$ th block row and $r$ th block column entry is

$$
\begin{aligned}
\frac{1}{N}\left(\mathcal{M}\left\{\mathbf{V}_{i}\right\} \mathcal{V}_{p}^{T}\right)_{q r} & =\int_{0}^{\theta} m_{n}^{(q)}(\tau) \\
& \left(\frac{1}{N} \sum_{j=k}^{N} \mathbf{v}\left(j T_{s}-\tau\right) \mathbf{v}^{T}\left((j+r-p) T_{s}\right)\right) d \tau
\end{aligned}
$$

Under the assumption of ergodicity, it is straightforward to show that (see (2))

$$
\begin{array}{r}
\lim _{N \rightarrow \infty} \frac{1}{N} \sum_{j=k}^{N} \mathbf{v}\left(j T_{s}-\tau\right) \mathbf{v}^{T}\left((j+r-p) T_{s}\right)= \\
\mathbf{R}_{\mathbf{v}} \delta\left(\tau,(p-r) T_{s}\right) .
\end{array}
$$

${ }^{7}$ The same approach is applied for $\mathcal{M}\left\{\mathbf{W}_{i}\right\}$.
Thus

$$
\begin{aligned}
& \lim _{N \rightarrow \infty} \frac{1}{N}\left(\mathcal{M}\left\{\mathbf{V}_{i}\right\}\right.\left.\mathcal{V}_{p}^{T}\right)_{q r}= \\
& \mathbf{R}_{\mathbf{v}} \int_{0}^{\theta} m_{n}^{(q)}(\tau) \delta\left(\tau,(p-r) T_{s}\right) d \tau .
\end{aligned}
$$

The problem of relation (40) is that the components of $\mathcal{M}\left\{\mathbf{V}_{i}\right\} \mathcal{V}_{p}^{T}$ can be bounded asymptotically. However, since $\tau$ belongs to $[0, \theta]$, requiring $(p-r) T_{s}>\theta$ guarantees that, for all $p, \delta\left(\tau,(p-r) T_{s}\right)$ equals zero and, by extension, $\lim _{N \rightarrow \infty} \frac{1}{N} \mathcal{M}\left\{\mathbf{V}_{i}\right\} \mathcal{V}_{p}^{T}=\mathbf{0}$. The condition $(p-r) T_{s}>\theta$ is unfortunately very conservative in practice. Thus, in general, reasonable values of $p$ in the past output scheme will lead to estimates of the system matrices with a bounded bias. A good way to circumvent this difficulty is to consider a classical bootstrapping approach, i.e. using noise free output data as instruments constructed from the simulation of a (biased) model estimated beforehand. This type of iterative scheme can be used to introduce optimal instrumental variable [29], [30], [31].

\section{SIMULATION EXAMPLE}

Consider the following 3rd order state space system

$$
\begin{aligned}
\dot{\mathbf{x}}(t) & =\left[\begin{array}{ccc}
0 & 1 & 0 \\
-3 & -2 & -1 \\
-1 & -2 & -1
\end{array}\right] \mathbf{x}(t)+\left[\begin{array}{ll}
1 & 1 \\
2 & 1 \\
1 & 2
\end{array}\right] \mathbf{u}(t) \\
\mathbf{y}(t) & =\left[\begin{array}{lll}
1 & 0 & 0 \\
0 & 0 & 1
\end{array}\right] \mathbf{x}(t)+\mathbf{v}(t) .
\end{aligned}
$$

coming from one of the demonstration program available in the CONTSID Toolbox. The input signal is made up with two independent pseudo random binary sequences of size $N=1000$. The sampling period is chosen equal to $0.1 s$. A white Gaussian noise is added on both outputs such that $^{8}$ the SNR $=20 \mathrm{~dB}$. The initial state vector is equal to zero. Monte Carlo simulation (MCS) analysis is used to evaluate the performance of the proposed estimation schemes (named RPM+MOESP and PMF+MOESP) which will be firstly compared to two other CT identification methods

- the PMF+GSVD algorithm [15] which combines the Poisson moment functionals and a subspace based method using a generalized singular value decomposition,

- the DT PI MOESP algorithm [19] leading to a discrete state space model converted into the CT domain with the $\mathrm{d} 2 \mathrm{c}$ function of Matlab.

For this first MCS of 100 runs, the instrumental variable is built up from past inputs (see (28)) with $p=4$. The user parameters of RPM+MOESP and PMF+MOESP are respectively fixed as $\hat{T}=25$ and $\lambda=\beta=3$. The same value of $\lambda$ is used for PMF+GSVD. The filter order is chosen as $i=n_{x}=3$. Figure 1 plots the estimated poles obtained via the considered algorithms. It clearly appears that the RPM+MOESP and PMF+MOESP techniques give relatively

${ }^{8} \mathrm{SNR}=10 \log \left(\frac{P_{y_{d}}}{P_{v}}\right)$ where $y_{d}$ is the deterministic part of $y$. 
good estimates of the complex poles but meet more difficulties to find accurately the real pole. It is however interesting to notice that the estimated real values are unbiased (centred around the expected one). On the contrary, the PMF+GSVD algorithm leads to biased values. Concerning the combination PI MOESP $+\mathrm{d} 2 \mathrm{c}$, the results are poor since the estimated poles are mistaken. Concerning PI MOESP $+\mathrm{d} 2 \mathrm{c}$, it is well known that the DT subspace algorithms need I/O Hankel matrices of relatively large size to guarantee good numerical properties. The requirement $i \geq n_{x}$ is also not sufficient. Simulations show that increasing $i$ improves the accuracy of the estimates. However, even in this case, the variance of the PI MOESP $+\mathrm{d} 2 \mathrm{c}$ estimated poles is quite significant. This first analysis shows that using CT subspace identification algorithms (with good user parameters values) can be considered as a good approach for the CT MIMO system identification.

The aim of the second MCS analysis is the study of the instruments choice influence on the poles estimation consistency. In addition to the instrumental variable proposed in this paper (see (28)), two other instrumental variable methods are considered

- a time shifted version of $\mathcal{M}\left\{\mathbf{U}_{i}\right\}$ with a time delay $\ell T_{s}=10 T_{s}$ and $i=3$ developed [12],

- a time derivative approximation input matrix $\mathcal{M}\left\{\mathbf{U}_{i}\right\}$ with $i=n_{u}+n_{y}+n_{x}+1$ used in the subspace algorithm proposed by R. Johansson et al as a correlation matrix (see [13] for details).

Figure 2 (in complement with figure 1) shows that, as expected, the use of relatively large number of time derivative inputs as instruments leads to poor numerical efficiency and produces less accurate estimates than considering time shifted inputs (see case 2 in fig. 2). Notice finally that the use of a time shifted version of $\mathcal{M}\left\{\mathbf{U}_{i}\right\}$ as instruments yields to estimated real poles with a bit larger variance than with $\boldsymbol{\Xi}=\mathcal{U}_{p}$

\section{CONCLUSION}

In this paper, the identification of CT MIMO state space models is considered. The proposed approach consists more precisely in introducing the reinitialised partial moments or the Poisson moment functionals to build a particular sampled I/O algebraic relationship from which a MOESP like algorithm can be applied. A specific attention has been concerned with the construction of the instrumental variable. This study, illustrated with a simulation example, has shown that choosing past sampled inputs as instruments leads to good performances, particularly in comparison with classical approaches using high order time derivatives.

\section{APPENDIX}

Consider the rhs of (25). Then, from (20), we asymptotically get

$$
\begin{aligned}
& \lim _{N \rightarrow \infty} \frac{1}{N} \mathcal{M}\left\{\mathbf{Y}_{i}\right\} \boldsymbol{\Pi}_{\mathcal{M}\left\{\mathbf{U}_{i}\right\}^{\perp}} \boldsymbol{\Xi}^{T} \mathbf{P}_{d}= \\
& \lim _{N \rightarrow \infty} \frac{1}{N}\left(\boldsymbol{\Gamma}_{i} \mathcal{M}\{\mathbf{X}\} \boldsymbol{\Pi}_{\mathcal{M}\left\{\mathbf{U}_{i}\right\}^{\perp}} \boldsymbol{\Xi}^{T}\right. \\
& +\mathbf{H}_{i} \mathcal{M}\left\{\mathbf{U}_{i}\right\} \boldsymbol{\Pi}_{\mathcal{M}\left\{\mathbf{U}_{i}\right\}^{\perp}} \boldsymbol{\Xi}^{T} \\
& \left.+\left[\mathbf{G}_{i} \mathcal{M}\left\{\mathbf{W}_{i}\right\}+\mathcal{M}\left\{\mathbf{V}_{i}\right\}\right] \boldsymbol{\Pi}_{\mathcal{M}\left\{\mathbf{U}_{i}\right\}^{\perp}} \boldsymbol{\Xi}^{T}\right) \mathbf{P}_{d} .
\end{aligned}
$$

By definition of the orthogonal projection

$$
\mathbf{\Pi}_{\mathbf{M}^{\perp}}=\mathbf{I}-\mathbf{M}^{T}\left(\mathbf{M M}^{T}\right)^{-1} \mathbf{M},
$$

the second term of the left-hand-side of the (42) vanishes as $N$ tends to infinity. Likewise, we have ${ }^{9}$

$$
\begin{gathered}
\mathcal{M}\left\{\mathbf{V}_{i}\right\} \boldsymbol{\Pi}_{\mathcal{M}\left\{\mathbf{U}_{i}\right\}} \boldsymbol{\Xi}^{T}=\mathcal{M}\left\{\mathbf{V}_{i}\right\} \boldsymbol{\Xi}^{T}-\mathcal{M}\left\{\mathbf{V}_{i}\right\} \\
\mathcal{M}^{T}\left\{\mathbf{U}_{i}\right\}\left(\mathcal{M}\left\{\mathbf{U}_{i}\right\} \mathcal{M}^{T}\left\{\mathbf{U}_{i}\right\}\right)^{-1} \mathcal{M}\left\{\mathbf{U}_{i}\right\} \boldsymbol{\Xi}^{T} .
\end{gathered}
$$

Since the instruments are chosen such as $\lim _{N \rightarrow \infty} \frac{1}{N} \mathcal{M}\left\{\mathbf{V}_{i}\right\} \boldsymbol{\Xi}^{T}=\mathbf{0}$, the first term of the rhs of the previous equation is asymptotically cancelled. The last problem concerns the second term of the rhs of (44) and more precisely $\frac{1}{N} \mathcal{M}\left\{\mathbf{V}_{i}\right\} \mathcal{M}^{T}\left\{\mathbf{U}_{i}\right\}$. In order to prove that this matrix product vanishes as $N$ tends to infinity, consider its $q$ th block row an $r$ th block column entry

$$
\begin{aligned}
& \frac{1}{N}\left(\mathcal{M}\left\{\mathbf{V}_{i}\right\} \mathcal{M}^{T}\left\{\mathbf{U}_{i}\right\}\right)_{q r}= \\
& \frac{1}{N} \sum_{j=1}^{N} M_{q}^{n}\left\{\mathbf{v}\left(t_{j}\right)\right\} M_{r}^{n T}\left\{\mathbf{u}\left(t_{j}\right)\right\} .
\end{aligned}
$$

By introducing the convolution products linked with the filters $M_{q}^{n}\{\bullet\}$ and $M_{r}^{n}\{\bullet\}$, we get

$$
\begin{aligned}
& \frac{1}{N}\left(\mathcal{M}\left\{\mathbf{V}_{i}\right\} \mathcal{M}^{T}\left\{\mathbf{U}_{i}\right\}\right)_{q r}=\int_{0}^{\theta} \int_{0}^{\theta} m_{n}^{(q)}(\tau) \\
& \quad\left(\frac{1}{N} \sum_{j=1}^{N} \mathbf{v}\left(t_{j}-\tau\right) \mathbf{u}^{T}\left(t_{j}-\mu\right)\right) m_{n}^{(r) T}(\mu) d \tau d \mu
\end{aligned}
$$

where $m_{n}^{(q)}$ and $m_{n}^{(r)}$ are defined as in equation (14) according to the chosen filtering method and

$$
\begin{aligned}
& \theta=\hat{T} \text { for the rpm } \\
& \theta=t_{j} \text { for the pmf. }
\end{aligned}
$$

Now, by ergodicity and since the disturbances are asymptotically independent of the input, $\lim _{N \rightarrow \infty} \frac{1}{N} \sum_{j=1}^{N} \mathbf{v}\left(t_{j}-\tau\right) \mathbf{u}^{T}\left(t_{j}-\mu\right)=\mathbf{0}$ and, by extension $\lim _{N \rightarrow \infty} \frac{1}{N} \mathcal{M}\left\{\mathbf{V}_{i}\right\} \mathcal{M}^{T}\left\{\mathbf{U}_{i}\right\}=\mathbf{0}$. All these steps prove the relation (26).

\footnotetext{
${ }^{9}$ The same approach is applied for $\mathcal{M}\left\{\mathbf{W}_{i}\right\}$.
} 

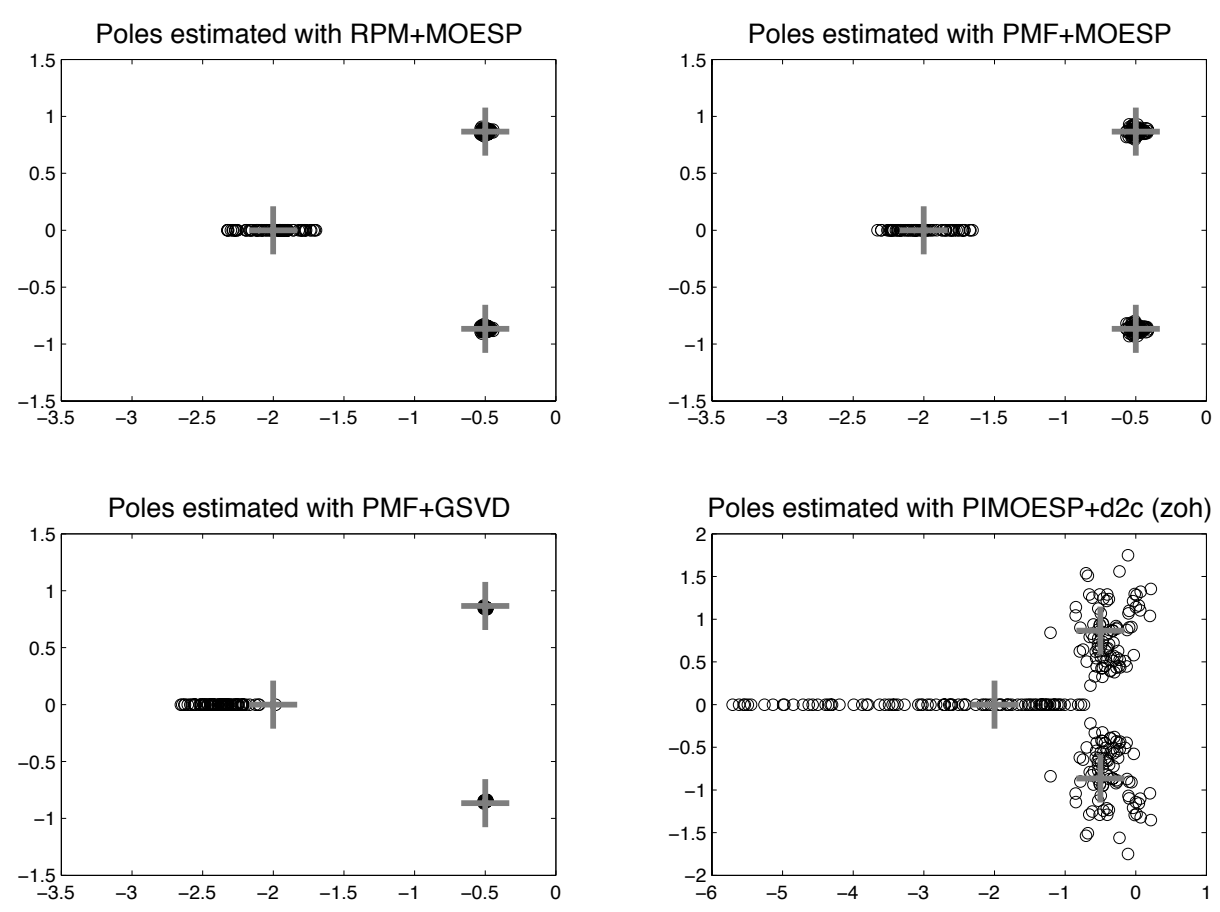

Fig. 1. Estimated poles obtained with RPM+MOESP, PMF+MOESP, PMF+GSVD and PI MOESP+d2c for $i=3$. The system poles are symbolized with grey crosses.
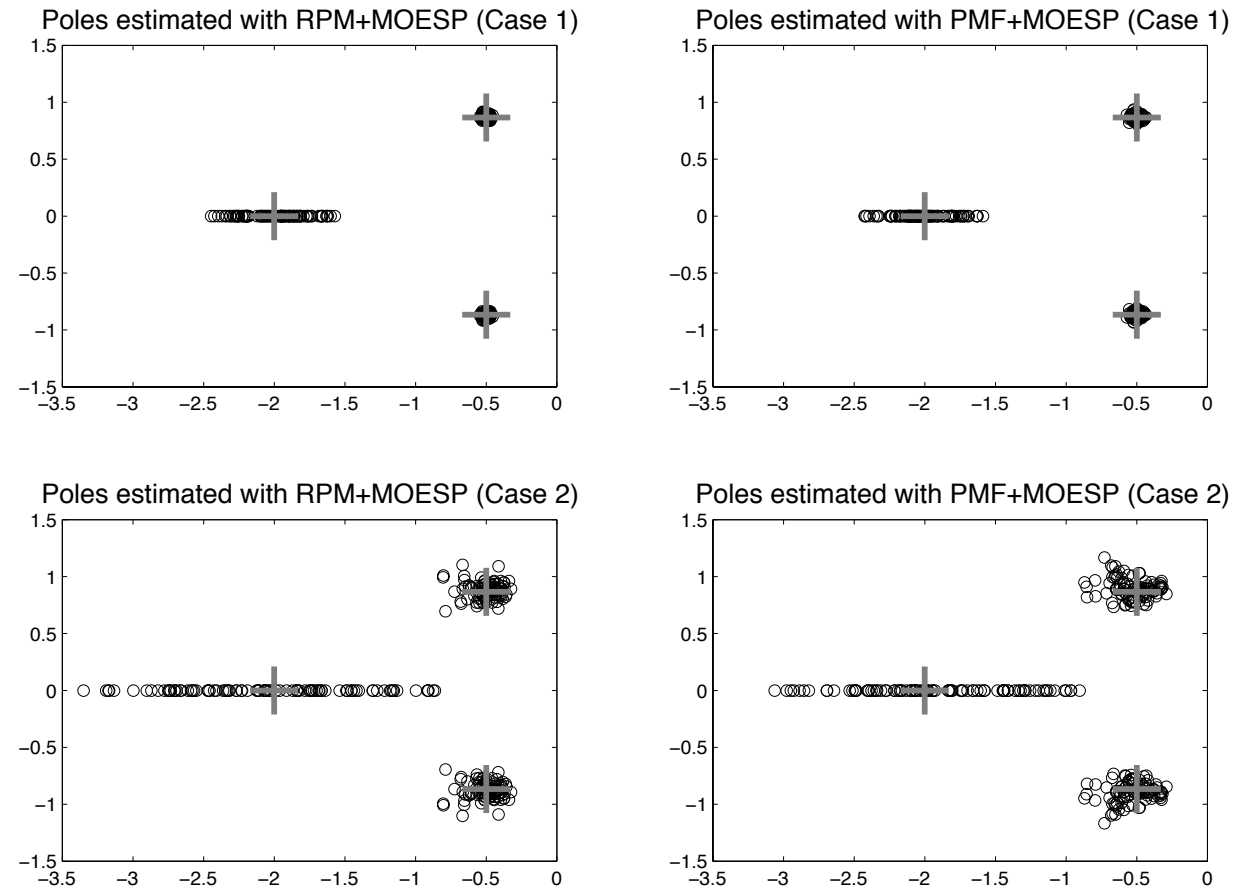

Fig. 2. Estimated poles obtained with RPM+MOESP and PMF+MOESP when the instrumental variable is built up from a time shifted version $(i=3$ and $\ell=10$ ) of $\mathcal{M}\left\{\mathbf{U}_{i}\right\}$ [12] (case 1) and from a filtered version of the inputs with $i=n_{u}+n_{y}+n_{x}+1$ [13] (case 2). 


\section{REFERENCES}

[1] P. Van Overschee and B. De Moor, Subspace identification for linear systems. Theory, implementation, applications. Kluwer Academic Publishers, 1996.

[2] T. Katayama, Subspace methods for system identification. Springer, 2005.

[3] L. Ljung, System identification. Theory for the user, 2nd ed. Upper Saddle River: PTR Prentice Hall Information and System Sciences Series. T. Kailath, Series Editor, 1999.

[4] M. Viberg, "Subspace based methods for the identification of linear time invariant systems," Automatica, vol. 31, pp. 1835-1851, 1995.

[5] D. Bauer, "Asymptotic properties of subspace estimators," Automatica, vol. 41, pp. 359-376, 2005.

[6] H. Unbehauen and G. Rao, "Continuous time approaches to system identification: a survey," Automatica, vol. 26, pp. 23-35, 1990.

[7] K. Aström, P. Hagander, and J. Sternby, "Zeros of sampled systems," Automatica, vol. 20, pp. 31-38, 1984.

[8] H. Garnier and L. Wang, Eds., Identification of continuous-time models from sampled data. To be published: Springer Verlag, 2007.

[9] H. Garnier, M. Mensler, and A. Richard, "Continuous-time model identification from sampled data: implementation issues and performance evaluation," International Journal of Control, vol. 76, pp. 1337 1357, 2003.

[10] M. Djamai, E. Tohme, R. Ouvrard, and S. Bachir, "Continuous time model identification using reinitialized partial moments. Application to power amplifier modeling," in 14th IFAC Symposium on System Identification, Newcastle, Australia, March 2006.

[11] D. Saha and G. Rao, Identification of continuous dynamical systems The Poisson Moment Functionals (PMF) approach. Berlin: Springer Verlag, 1983.

[12] B. Haverkamp, C. Chou, M. Verhaegen, and R. Johansson, "Identification of continuous time MIMO state space models from sampled data in the presence of process and measurement noise," in 35th Conference on Decision and Control, Kobe, Japan, December 1996.

[13] R. Johansson, M. Verhaegen, and C. Chou, "Stochastic theory of continuous time state space identification," IEEE Transactions on Signal Processing, vol. 47, pp. 41-51, 1999.

[14] B. Haverkamp, "State space identification: theory and practice," Ph.D dissertation, Delft University of Technology, The Netherlands, 2001.

[15] T. Bastogne, H. Garnier, and P. Sibille, "A PMF-based subspace method for continuous-time model identification. Application to a multivariable winding process," International Journal of Control, vol. 74, pp. 118-132, 2001.

[16] A. Ohsumi, K. Kameyama, and K. Yamagushi, "Subspace identification for continuous time stochastic systems via distribution based approach," Automatica, vol. 38, pp. 63-79, 2002.

[17] W. Li, H. Raghavan, and S. Shah, "Subspace identification of continuous time models for process fault detection and isolation," Journal of Process Control, vol. 13, no. 5, pp. 407-421, 2003.

[18] M. Verhaegen and P. Dewilde, "Subspace model identification part 1: output error state space model identification class of algorithms," International Journal of Control, vol. 56, pp. 1187-1210, 1992.

[19] — - "Subspace model identification part 2: analysis of the elementary output error state space model identification algorithm," International Journal of Control, vol. 56, pp. 1211-1241, 1992.

[20] M. Verhaegen, "Subspace model identification part 3: analysis of the ordinary output error state space model identification algorithm," International Journal of Control, vol. 58, pp. 555-586, 1993.

[21] — - "Identification of the deterministic part of MIMO state space models given in innovations form from input output data," Automatica, vol. 30, pp. 61-74, 1994.

[22] R. Johansson, "Identification of continuous time models," IEEE Transactions on Signal Processing, vol. 42, pp. 887-897, 1994.

[23] H. Garnier, M. Gilson, and O. Cervellin, "Latest developments for the Matlab CONTSID toolbox," in 14th IFAC Symposium on System Identification, Newcastle, Australia, March 2006.

[24] P. Young, "Parameter estimation for continuous time models: a survey," Automatica, vol. 17, pp. 23-39, 1981

[25] A. Jemni and J. Trigeassou, "A comparative study of the deterministic accuracy of two identification methods for continuous time systems," in IEEE SMC CESA'96 IMACS Symposium on Modelling, Analysis and Simulation, Lille, France, July 1996

[26] M. Jansson and B. Wahlberg, "On consistency of subspace methods for system identification,” Automatica, vol. 34, pp. 1507-1519, 1998.
[27] I. Goethals, "Subspace identification for linear, Hammerstein and Hammerstein Wiener systems," Ph.D. dissertation, Katholieke Universiteit Leuven, Faculteit der Toegepaste Wetenschappen, 2005.

[28] D. Bauer, "Order estimation for subspace methods," Automatica, vol. 37, pp. 1561-1573, 2001

[29] P. Stoica and T. Söderström, "Optimal instrumental variable methods for identification of multivariable linear systems," Automatica, vol. 19, pp. 425-429, 1983.

[30] M. Gilson and P. Van den Hof, "Instumental variable methods for closed loop system identification," Automatica, vol. 41, pp. 241-249, 2005.

[31] M. Gilson and G. Mercère, "Subspace based optimal IV method for closed loop system identification," in 14th IFAC Symposium on System Identification, Newcastle, Australia, March 2006. 